\title{
Giant Bleeding Pseudo-aneurysm of left Sub-clavian Artery Complicating Neurologic Impairment: Management Challenges (Case Report)
}

\author{
Nwafor Ikechukwu Andrew*, Emeruem Nwadinma Uloma, Onyia Ugochukwu Obinna Chibuife, \\ Eze John Chukwuemeka, Okorie Chukwuemeka Ogueri
}

National Cardiothoracic Center of Excellence, University of Nigeria Teaching Hospital, Ituku-Ozalla, Enugu, Nigeria

Email address:

ikechukwu.nwafor@unn.edu.ng (N. I. Andrew)

*Corresponding author

\section{To cite this article:}

Nwafor Ikechukwu Andrew, Emeruem Nwadinma Uloma, Onyia Ugochukwu Obinna Chibuife, Eze John Chukwuemeka, Okorie Chukwuemeka Ogueri. Giant Bleeding Pseudo-aneurysm of left Sub-clavian Artery Complicating Neurologic Impairment: Management Challenges (Case Report). International Journal of Cardiovascular and Thoracic Surgery. Vol. 7, No. 2, 2021, pp. 16-19.

doi: $10.11648 /$ j.ijcts. 20210702.12

Received: April 12, 2021; Accepted: April 23, 2021; Published: May 26, 2021

\begin{abstract}
Subclavian artery pseudoaneurysms are rare and occur mostly as a consequence of an inadvertent arterial puncture during central venous catheterization, endovascular therapeutic procedures or after penetrating or blunt trauma. They usually have a late clinical presentation, with pain, swelling or other compressive symptoms. We present a 40 -year old man farmer who presented to our service with prior 14 days and 12 days history of left chest upper chest swelling and inability to use the left upper limb respectively, all on account of injury. He went on a night alcohol binge, got drunk and while getting to his sitting room slipped and fell on a nearby glass center table. He had a deep cut on the left upper anterior chest wall. There was immediate profuse spurting bleeding with estimated blood loss of 800 ml. After delay in definitive treatment due to financial constraint the aneurysm increased in size, ruptured and rebled profusely leading to syncope. He was managed by Doppler ultrasound, median sternotomy and subclavian artery exploration to achieve proximal and distal vascular control. Sac was entered into via an infraclavicular transverse incision, heamatoma manually evacuated, bleeding site isolated and secured. He received 5 units of blood. Postoperative course was uneventful as he was managed with analgesics, antibiotics, haematinics and physiotherapy. Power in the upper limb has improved to 3 around the shoulder, 2 around the elbow and 1 around the wrist.
\end{abstract}

Keywords: Giant, Bleeding, Aneurysm, Left Subclavian Artery, Brachial Plexus

\section{Introduction}

Pseudo-aneursysms of the subclavian artery are rare but present a surgical challenge because of the complex anatomy and accompanying collateral damage during the repair. Anatomically, the subclavian artery is divided into 3 parts by scalenus anterior muscle with vertebral, internal mammary and thryocervical trunk branches arising from the first part while the costoclavicular and dorsal scapular branches arise from the second and third part respectively $[1,2]$. Subclavian artery pseudoaneurysms impose a major surgical challenge, especially when originating from the proximal third. Large pseudoaneurysms may rupture or produce signs and symptoms of compression. If intervention is considered necessary, several options are available: open surgical resection and vascular reconstruction, endovascular exclusion, stent graft implantation or ultrasound-guided thrombin injection have all been described. The choice of procedure should be tailored to the patient, based on comorbidities, clinical presentation and anatomic characteristics. When compressive symptoms exist, an open approach is advised. However, because of their location, surgical exposure of the pseudoaneurysm may be technically difficult, requiring a sternotomy or a clavicular resection for adequate exposure. An endovascular approach demands an 
adequate landing zone and absence of severe tortuosity $[3,4]$. SCA2.

\section{Case Summary}

We present a 40-year old man farmer who presented to our service with prior 14 days and 12 days history of left chest upper chest swelling and inability to use the left upper limb respectively, all on account of injury. He went on a night alcohol binge, got drunk and while getting to his sitting room slipped and fell on a nearby glass center table. He had a deep cut on the left upper anterior chest wall. There was immediate profuse spurting bleeding with estimated blood loss of $800 \mathrm{mls}$. He became weak and dizzy. Bleeding was arrested with pressure dressing before being taken to a nearby hospital. He was resuscitated with intravenous normal saline and was given antibiotics, analgesics and haematinics.

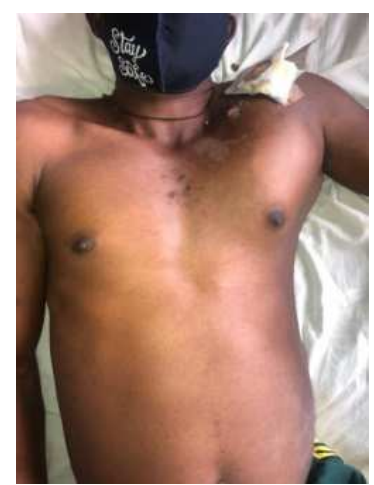

Figure 1. Showing the patient with Left anterior chest swelling.

He defaulted referral to our service for definitive management and went home. While at home the swelling increased in size with associated severe chest pains. In addition, there was weakness in the left upper limb. Ten days after initial hospitalization, the weakness in the limb worsened and swelling increased in size leading to rupture and rebleeding. He lost consciousness and was rushed to the same hospital and was transfused with two units of blood and wound redressed with pressure packing and sent to our service for definitive treatment.

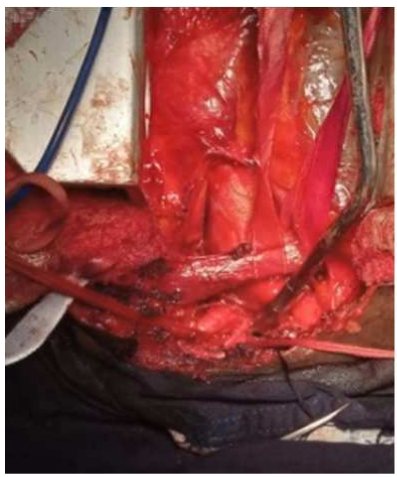

A

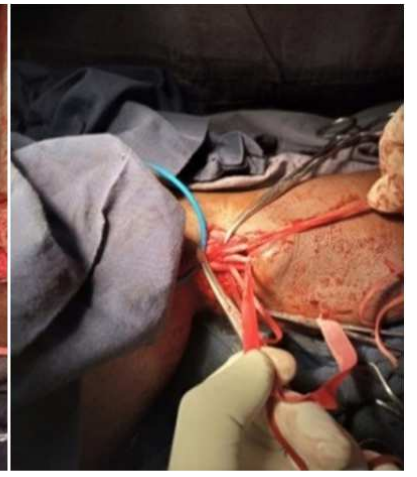

B
Figure 2. Innominate vein and innominate artery being displayed enroute to isolating and securing the sub-clavian artery B. axillary artery being isolated.
At our service, the above details were noted. He was fairly stable with blood pressure of $100 / 60 \mathrm{mmHg}$, Pulse rate of 98 per minute, low volume but regular rhythm. Chest examination revealed a mass of $12 \times 16 \times 5 \mathrm{~cm}$ occupying the anterior left upper chest, extending from the clavicle to the axilla and the arm with infraclavicular sutured wound oozing seropurulent fluid. The mass was pulsatile with a bruit. Left upper limb evaluation showed grade zero power across all muscle groups: shoulder, elbow and wrist. There was hypotonia across all joints. Sensation was however intact. The left radial artery was however present though of small volume. A working diagnosis of ruptured pseudoaneurysm of left subclavian artery with brachial plexus nerve branches impairment was made, He was optimized for emergency surgery, receiving another two units of blood. He had median sternotomy and axillary artery exploration for both proximal and distal vascular control.

Intraoperative findings were huge haematoma sac $(20 \times 15$ $x$ 8) $\mathrm{cm}$ with massive blood clots and rent in the third part of left subclavian artery and a piece of broken glass within the sac. Blood clots were manually evacuated with profuse bleeding which was controlled with digital pressure and vascular clamps. Efforts to isolate and suture the bleeding points failed due to distorted anatomy. Consequently the tissues bearing the bleeding site were transected and proximally and distally ligated. The digital pulse oximeter however was $95-97 \%$. He received 5 units of blood intraoperatively. Wounds were closed in layers with mediastinal and pericardial drains as well as redivac wound drain of the haematoma cavity.

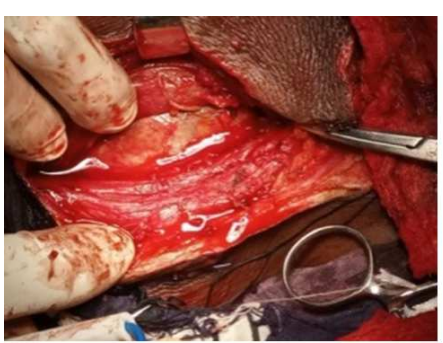

A

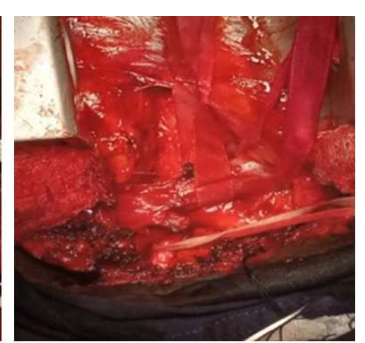

B
Figure 3. The aneurysmal sac being displayed before it was entered into and the clot evacuated.

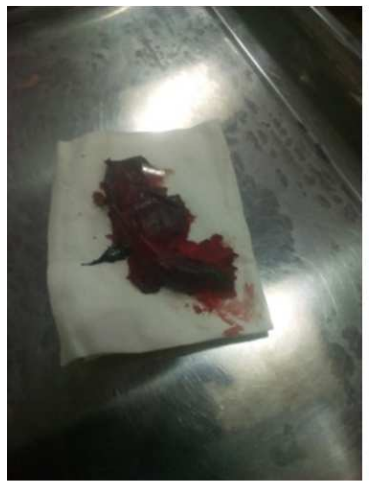

A

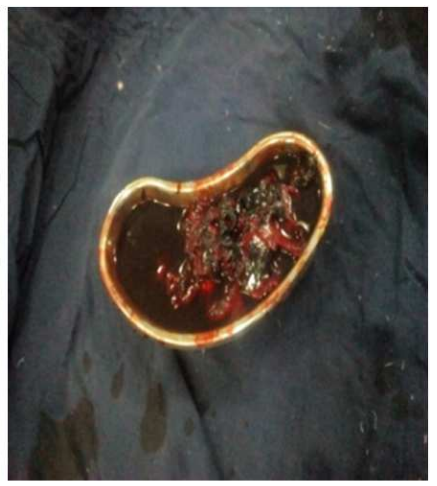

B
Figure 4. Showing the aneurysmal sac and the evacuated clots. 
Immediate postoperative conditions were uneventful as he was managed with analgesics, antibiotics, haematinics and physiotherapy. On the $7^{\text {th }}$ postoperative day, it was discovered that he had superficial site infection around the haematoma cavity site and was managed with daily dressing till healing was achieved. He has continued physiotherapy on outpatient basis and the power in the upper limb has improved to 3 around the shoulder, 2 around the elbow and 1 around the wrist.

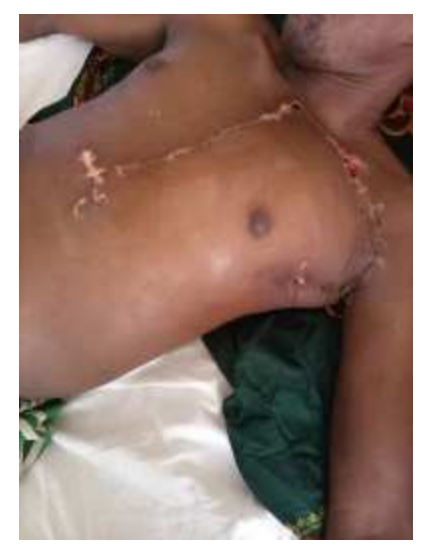

Figure 5. Showing the patient on the $7^{\text {th }}$ postoperative day with healing surgical site infected wounds.

\section{Discussion}

Extra-thoracic SAAs most commonly present as tender pulsatile masses in the superior fossa, and Horner's syndrome including brachial plexopathy can also be a presentation of an extra-thoracic arterial aneurysm [5, 6]. In the index patient, the weakness and loss of power in the left upper limb worsened as the aneurysm increased in size, showing compression effects.

Surgery is the main treatment for SAAs. [5] Location of the aneurysm determines the surgical approach taken. For extrathoracic SAAs, supraclavicular incision is adequate for aneurysmectomy. However, in the index case, the size of the aneurysm precluded supraclavicular approach as that might have led to inadvertent entry into the aneurysmal sac prior to proper vascular control. Lateral thoracotomy is preferred for the left intrathoracic SAAs. [7]. Again, the size of the aneurysm precluded this treatment approach to get proximal control as retracting the ribs would have been difficult on account of the size of the aneurysm.

The vast majority of the subclavian artery lesions are the result from penetrating trauma, and approximately $25 \%$ of these injuries are caused by blunt trauma. It has been described an overall mortality rate around $60 \%$ in patients that did not make it into the hospital because they died during transportation or at the crash site. The hospital mortality rate 'is about 5 to $30 \%$ in the patients that survived the triggering event [8]. Despite the delay in seeking definitive treatment, the index patient survived because the rent in SCA was about $1 \mathrm{~cm}$ according to the Doppler ultrasound. Patient could not afford the cost of CT angiography. He received a total 9 units of blood in the course of management.
Recently, as a less invasive alternative to surgical repair, endovascular stent-graft treatment has become possible. [9]. However, experience with the use of stent's for SAA's remains limited because of its relative infrequency. [10]. In low-income settings like ours without technical know-how and requisite equipment, expertise in open approach becomes indispensable to save life as in the index case.

In our center there have been previous reports of all types of extracranial artery aneurysms, with challenges of management well highlighted [11-13].

\section{Conclusion}

The challenges of managing a giant bleeding pseudoaneurysm of left SCAA with resultant compression of brachial plexus in a low-income setting like ours are 1. Inability of the patient to pay for treatment expenses due to absence of functional health insurance 2. Non-availability of expertise and equipment in minimal access technique and 3. Dysfunctional national blood banks for altruistic blood donations. Studies comparing open repair with stenting show different kinds of complications but a comparable complication rate. However, long-term results show a better patency rate during open repairs.

\section{References}

[1] Vvyas JM, Subramman RK, Kumar SKS, Branching Pattern of Subclavian Artery: Cadavericc Study. Journal of Clinical and Diagnostic Research 2018; 12 (12): AC11-AC12.

[2] Yuca AH, Kizilkanat E, Ocdemir CO, Variations of the subclavian artery and its branches. Okajimas Folia Anatomica Japonica 1998; 76 (5): 255-262.

[3] Ferreira RS, Castro JM, Concalves FB, Moreu R, Correla R, Rodriguez R et al. Surgical Management of Subclavian Artery Pseudoaneurysm: A case report and literature. Rev Port Cir Cardiovascular Surg 2017; 24 (3-4): 105-106.

[4] Asai M, Van Houtte O, Sullivan TR, Garrido M, Pineda DM. Endovascular Repair of Three Concurrent Mycotic Pseudoaneurysm. Vasc Endovasc Surg 2016; 52 (6): 473-477. Doi: $1177 / 1538574418772458$.

[5] bDavidovic LB, Markovic DM, Pejkic SD, Kovacevic NS, Colic MM, Doric PM. Subclavian artery aneurysms. Asian J Surg. 2003; 26: 7-11

[6] Utikal P, Bachleda P, Kocher M, Novotny J, Drac P, Drac P. Aneurysm of the subclavian artery. Acta Univ Palacki Olomuc Fac Med. 1999; 142: 107-9.

[7] Dougherty MJ, Calligaro KD, Savarese RP, DeLaurentis DA. Atherosclerotic aneurysm of the intrathoracic subclavian artery: A case report and review of the literature. J Vasc Surg. 1995; 21: 521-9.

[8] Enamorado-Enamorado J, Guerrero JJ, Revuelto-Rej J, Gordillo-Escobar E, Herreira-Metero C. Left Subclavian Artery Pseudoaneurysm aftea Traffic Accident: A Case Report. Hindawi Publishing Corporation Case Reports in Critical Care Volume 2011, Article 451819, 2 pages $\backslash$ doi: $10.1155 / 2011 / 451819$. 
[9] Cires G, Noll RE, Jr, Albuquerque FC, Jr, Tonnessen BH, Sternbergh WC., 3rd Endovascular debranching of the aortic arch during thoracic endograft repair. J Vasc Surg. 2011; 53: 1485-91.

[10] Hernandez JA, Pershad A, Laufer N. Subclavian artery pseudoaneurysm successful exclusion with a covered selfexpanding stent. J Invasive Cardiol. 2002; 14: 278-9.

[11] Nwafor IA, Eze JC, Ezemba N, Anyanwu CH. The challenges faving the management of arterial aneurysms in UNTH, Enugu, Nigeria. Nig J of Med 2013; 21 (4): 438-40.

[12] Nwafor IA, Eze JC, Ezemba N, Ngene CI, Akpan AF, Giant pseudoaneurysm of a splanchnic artery; A Case Report. Nig J Med 2015; 24 (3): 268-272.

[13] Eze JC, Ezemba N, Adamu Y. A study of Extracranial Artery Aneurysm at UNTH, Enugu. Nig J Clin Practice 2010; 13 (3): 272-5. 